\title{
HRTEM Based SADP Image Indexing Using Image Processing
}

\author{
${ }^{1}$ Dibyendu Roy, ${ }^{2}$ Niladri Halder, ${ }^{3}$ Poushali Roy \\ ${ }^{1,2,3}$ Electronics and Communication Engineering , Department UIT Burdwan, University Institute of Technology, \\ Burdwan University
}

\begin{abstract}
In this paper firstly the input images (which are a SADP of Ni Ti based thin film taking using HRTEM) have been segmented out from the background and clearly get the six rings patterns. Using HRTEM instrument the selected area diffraction pattern (SADP) of the film have been obtained. Then these rings are indexed carefully. To index a selected area diffraction pattern it is necessary to assign miller indices, \{hkl\} to each ring. It can be done by determining the ring diameter and then comparing the ratio of radius with the inter planner spacing. Another process of indexing has been done from the peak positions of x-ray diffraction pattern. Keywords: MEMS sample, HRTEM, SADP indexing, crystal plane determination.
\end{abstract}

\section{Introduction}

MEMS stand for micro electro mechanical system. Basically MEMS are small integrated device or structure that combines electrical and mechanical components. The typical size of MEMS is 1- $100 \mu \mathrm{m}$ like human hair thickness $(50 \mu \mathrm{m})$, red blood cells $(10 \mu \mathrm{m})$ etc. Materials like polymers, metals, ceramics, and silicon have been used as MEMS material. Here Ni Ti shape memory alloy have been used as MEMS sample. Ni Ti based alloys has been chosen because it is a practical shape memory alloy with high strength and ductility [1-5]. It also has the property like pre-transformation behaviors. Depending on how good eyes are, the smallest distance between two points that can be resolve with human eyes is about $0.1-0.2 \mathrm{~mm}$, is called the resolution or resolving power of human eyes. So any instrument that can show pictures or images revealing detail finer than $0.1 \mathrm{~mm}$ is known as a microscope and its highest magnification is governed by its resolution. Transmission electron microscope (TEM) can continuously gives information in real space and reciprocal space. This instrument has been used because using TEM both the diffraction pattern and the image of the specimen from the common part can be obtained. High resolution means ability to see two closely spaced features in the sample as distinct. HRTEM is an instrument for high-magnification studies of nano materials. High resolution makes it accurate for imaging materials on the atomic scale. HRTEM is a phase-contrast imaging technique, which makes it able to obtain images with atomic resolution. It can be used to investigate the crystalline structure of the sample, including identification of lattice planes and some defects. It is a crystallographic tool that can be used to identify crystal phases and orientations [6-8].

Here images of selected area diffraction pattern of a $\mathrm{Ni} \mathrm{Ti}$ based thin film have been taken using HRTEM which is shown in figure 1.

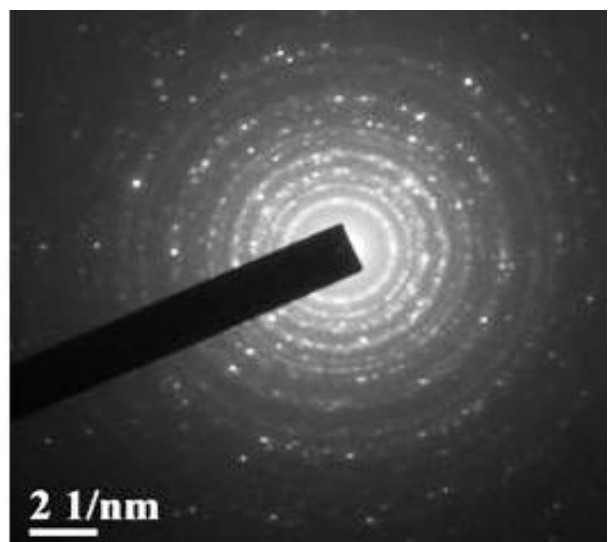

Figure 1: Selected area diffraction pattern of HRTEM of a Ni Ti based thin film.

Ni Ti shape memory alloy also known as 'Nitinol' alloys. This 'Nitinol' alloys have been made amorphous over a wide composition range by sputter deposition ion implantation and electron irradiation technique etc. These alloys have very important properties for medical and technical application. Images and selected area diffraction pattern (SADP) of the sputtered and ion implanted sample [9-11] were recorded on photographic film with 120 or $200 \mathrm{kv}$ TEMs. Material image processing is the basic step to analyse images and 
extract data from them. Basically image segmentation is an important part of material image processing. Using image segmentation process an image have been cluster into different disjoint parts by groping the pixels to form a region of homogeneity based on the pixel characteristics like colour, gray level, texture, intensity and many other features. The main goal of image segmentation is to clearly differentiate the object from the background in an image [12-13]. The simplest process of image segmentation is the thresholding. It helps to enhance and detect the object from the original image. In thresholding process each pixel in an image is represented by some black and white pixel. Some fixed value has been considered as the threshold value. Now if the image intensity is less than the fixed threshold value then that pixel will be black otherwise it will be white pixel [14]. Image enhancement is the process in which the more suitable and clear image than the original one can be obtained. Actually enhancement of image is the process of sharpening the image feature by intensity and saturation transformations, contrast enhancement, edge enhancement, slicing, producing synthetic stereo image, making digital mosaics etc [15-16]. There are various noise removal techniques are available in image processing. Depending on the type of the noise the appropriate noise removal technique have been chosen. It is very important to choose what type of filter should be use to remove what type of noise [17-18]. When noise added with the original image thresholding process becomes very complex so that the right threshold value cannot be obtained. Binarization is the process of finding the appropriate threshold value [19-21]. Every crystalline solid has its own unique characteristics. Actually structure determination is the process of knowing how the atoms pack together in the crystalline state and what is inter atomic distance, what is the angle etc. The shape and size of the unit cell for any compound can easily be determined. This is called indexing. Basically indexing is the process of obtaining crystallographic data from a specimen of material [22-25].

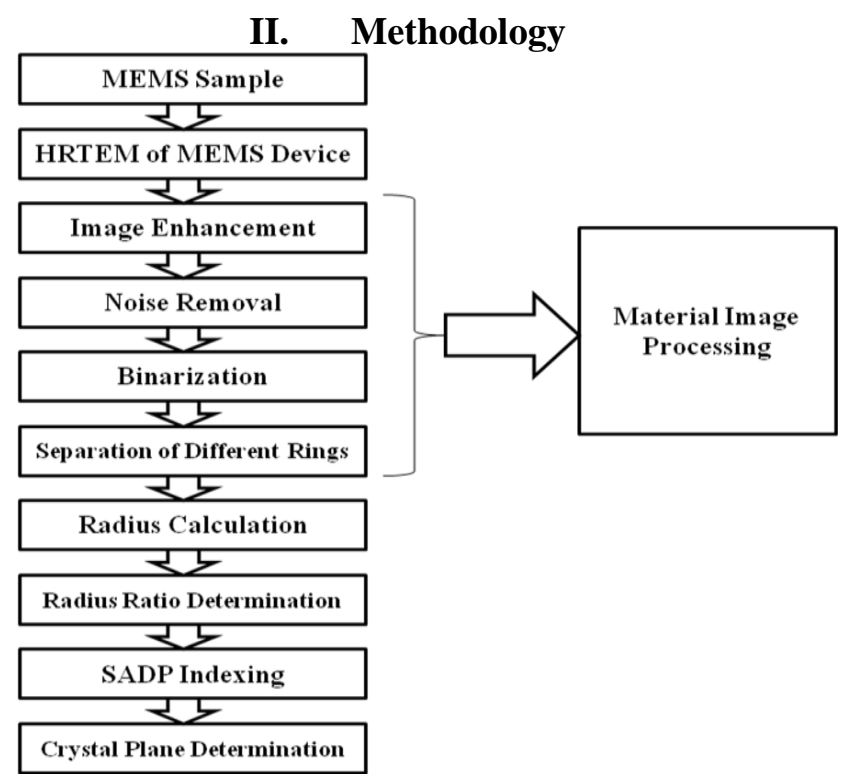

Figure 2: Flow chart of the total process from MEMS sample to crystal plane determination.

\subsection{Material image processing}

Material image processing is the process that describes any images of any material in so details that can investigate and analyse the material in molecular scale. Using this process an object can be separated from other in an image, also the shape, size and colour of the original image [12-13] can be changed. This process combines different image processing task like image enhancement, noise removal, binarization, separation of different ring patterns etc.

\subsection{Image Enhancement}

Image enhancement is use as pre-processing tools in image processing technique. Image enhancement is the process in which the more suitable and clear image than the original one has been obtained. It is a very easy process. For example it can be said that if the image may lighter or darken, increase or decrease the contrast then the processed image become more suitable and accurate than the original one. This is also known as enhancement of image. Actually enhancement of image is the process of sharpening the image feature by intensity and saturation transformations, contrast enhancement, edge enhancement, slicing, producing synthetic stereo image, making digital mosaics etc. As a result the selected feature can be easily detected from the original image [15-16]. Image enhancement alters the image impact on the viewer. In image enhancement process the restoration process is very important because it distorts the original digital values. 


\subsection{Noise Removal}

It is known to all that any unwanted information present in a data is called noise. Noise has very vital impact on the image processing technique. In an image noise may added from many sources. An image may contain speckles, stains, dots etc. These are called noise. In image processing technique the digital image converts the optical signal into electrical signal and finally into digital signal. In this conversion process noise easily enter in the digital image. So noise removal is very important task in material image processing [17-18]. There are various noise removal techniques are available in image processing. Depending on the type of the noise the appropriate noise removal technique have been chosen.

\subsection{Binarization}

The simplest process of image segmentation is the thresholding. It helps to enhance and detect the object from the original image [14]. But when there is a noise with the original image then the thresholding process become very complex. If the right threshold value cannot be selected then the detection of the correct pixel value of the background cannot be done. As a result there will be mismatch and degradation in the total system performance. So it is very important to choose the correct threshold value. Binarization is the process of finding the appropriate threshold value. There are several methods of image binarization such as entropy method [19], minimum error method, means threshold method, analysis method etc [20-21].

\subsection{Separation of Different Ring Patterns}

When the binarization has been completed, the correct threshold value has been chosen and enhances the rings in the original image. Then the separation of those rings using some image processing software becomes very easy. After this separation of these rings each ring appears more specifically. Since each ring become more specific than in the original image after separation, the next experimental task become very easier. So easily the ring diameter has been measured. As a result the indexing process of those ring become simple. The segmented rings are shown in the figure 3 .
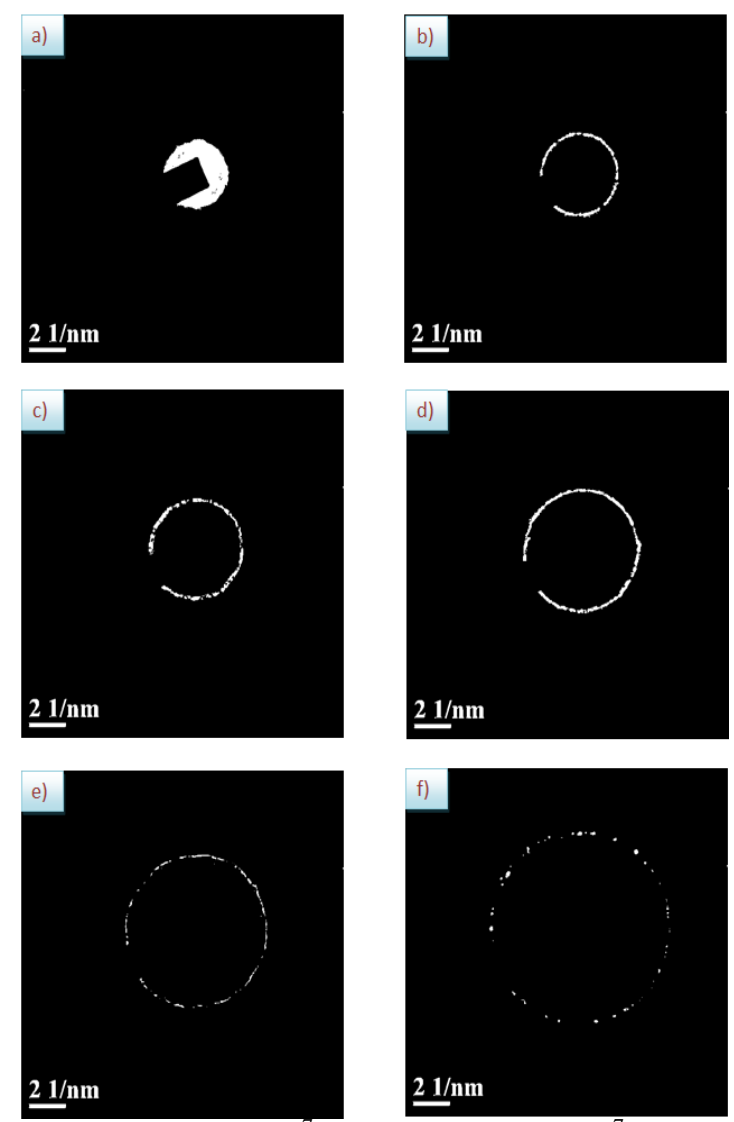

Figure3: Separated rings are: (a) radius $1.5 \times 10^{7} \mathrm{~nm}$ (b) radius $1.7 \times 10^{7} \mathrm{~nm}(\mathrm{c})$ radius $2 \times 10^{7} \mathrm{~nm}$ (d) radius $2.5 \times 10^{7} \mathrm{~nm}$ (e) radius $3 \times 10^{7} \mathrm{~nm}$ (f) radius $3.8 \times 10^{7} \mathrm{~nm}$.

In case of ring pattern the diameter of each ring is the characteristic of the spacing of the reflecting planes in the crystal. At first the determination of the centre of those concentric circles has been done. Then the measurement of the ring diameter using some image processing technique has been done. Actually the 
difference between two diameter of two successive ring is the inter planner spacing which is denoted by d. As the rings diameters are measured our next step is to indexing the rings.

\subsection{SADP Indexing}

Selected area diffraction pattern (SADP) is employed to select the interest of specimen to obtain its diffraction pattern that is projection of the reciprocal lattice of interest. In SADP, the brightest spot usually appears in the centre of the diffraction pattern. Basically one projected SADP can provide two dimensional crystallographic data, like two axes of the lattice parameters. To obtain complete crystallographic information it is necessary to tilt the sample to obtain a series of SADPs [9-11]. There are different methods of indexing SADPs. These are "comparing the ratio of ring diameter with the inter planer spacing", "start with zone axis" and "start with diffraction pattern" etc.

Indexing is the process of determining the unit cell dimension. It can be done by determining the ring diameter and then comparing the ratio of radius with the inter planner spacing. Another process of indexing is done from the peak positions of x-ray diffraction pattern. It is not just the simple reverse of calculating peak positions from the unit cell dimension and wavelength. To index a powder diffraction pattern it is necessary to assign miller indices, $\{\mathrm{hkl}\}$. Indexing is always the first step of analysis [22-25]. A diffraction pattern cannot be analyzed until it has been indexed. Basically indexing is the process of obtaining crystallographic data from a specimen of material.

\section{Result \& Discussions}

The experimental thin film material is $\mathrm{Ni} \mathrm{Ti}$. Now the following procedure have been followed to index the ring pattern -

a) Firstly the ring diameters have been measured. Suppose $R_{n}$.

b) Then determinations of the square of diameters of the outer rings to that of the first or second ring have been done.

Suppose the square of diameters of the outer rings to that of the first ring is $\left(R_{n} / R_{1}\right)^{2}$. Where $R_{n}$ is the diameter of the outer most ring and $\mathrm{R}_{1}$ is the diameter of the inner most ring.

c) Finally the ratios against the table of ratios of the inter planner spacing for the crystal structure of interest have been checked.

Comparing the ratios against the table of ratios of the inter planner spacing for the crystal structure of interest it can be decided that the crystal under study is simple cubic crystal. The indexed separated rings are shown in figure 4 .
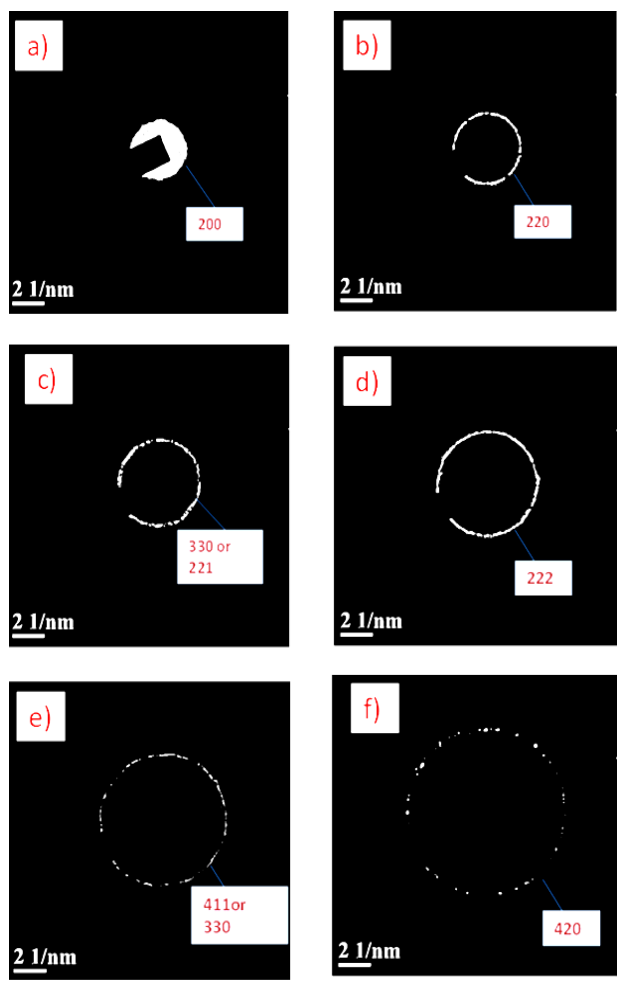

Figure 4: Indexed separated rings are: (a) radius $1.5 \times 10^{7} \mathrm{~nm}-200$ (b) radius $1.7 \times 10^{7} \mathrm{~nm}-220$ (c) radius $2 \times 10^{7}$ $\mathrm{nm}-330$ or 211 (d) radius $2.5 \times 10^{7} \mathrm{~nm}-222$ (e) radius $3 \times 10^{7} \mathrm{~nm}-411$ or 330 (f) radius $3.8 \times 10^{7} \mathrm{~nm}-420$. 
Now the indexed ring pattern of the selected area diffraction pattern of HRTEM of a Ni Ti based thin film have been presented which is shown in figure below.

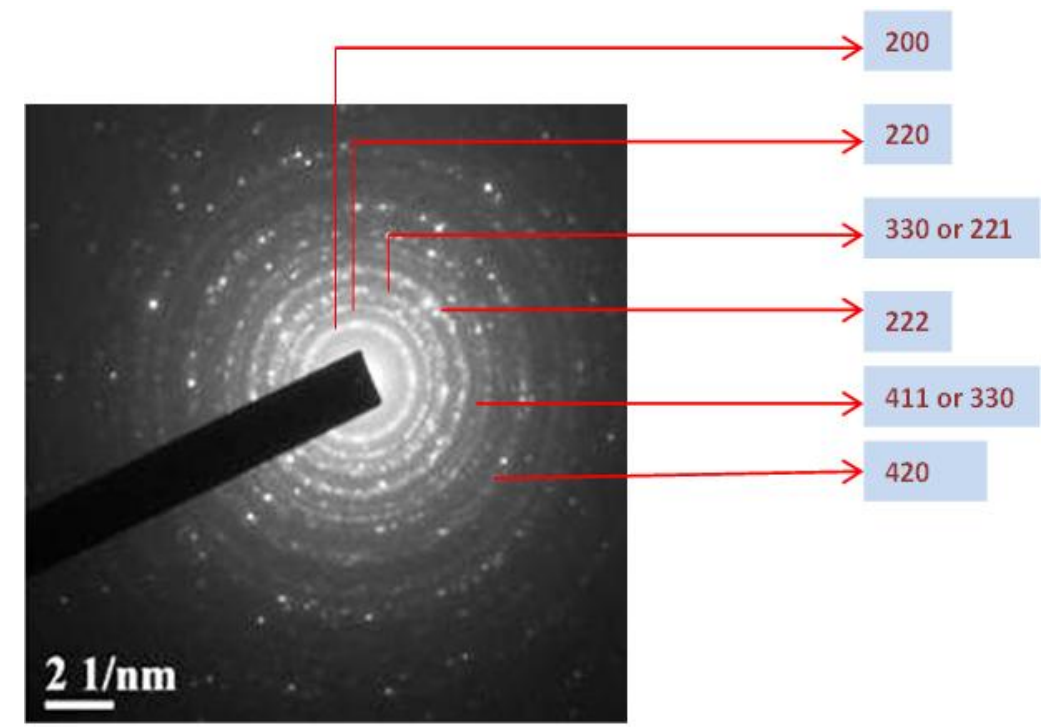

Figure 5: Indexed selected area diffraction pattern of HRTEM of a Ni Ti based thin film.

\section{Conclusion}

Using HRTEM the SADP of thin film have been obtained. There is no requirement of any XRD pattern. In this project firstly the rings from the original image are segmented. Then six rings from the original image have been obtained. Finally the ring patterns are indexed using the method of comparing the square of ratios of the ring diameter with the inter planner spacing d. Finally indexed the rings as $1^{\text {st }}$ ring $200,2^{\text {nd }}$ ring 220 , $3^{\text {rd }}$ ring 330 or $221,4^{\text {th }}$ ring $222,5^{\text {th }}$ ring 411 or $330,6^{\text {th }}$ ring 420 from centre to outer direction respectively have been done.

Special thanks to IIT Kharagpur, West Bengal, India.

\section{Acknowledgments}

\section{References}

[1]. Journal of Micro/Nanolithography, MEMS, and MOEMS Chris A. Mack, Lithoguru.com, Editor-in-Chief.

[2]. Hoo-Jeong Lee and Ainissa G. Ramireza(2004), Crystallization and phase transformations in amorphous NiTi thin films for microelectromechanical systems, APPLIED PHYSICS LETTERS VOLUME 85, NUMBER 7.

[3]. Wilcox, D.L; and Howell, L.L. Fully compliant tensuralbistable micro-mechanisms (FTBM). Journal of Micro-electromechanical Systems 2005,14 (6), 1223-1235.

[4]. Madou, M.J.; Fundamentals of Micro-fabrication: the Science of Miniaturization; CRC Press: New York, NY, 2002.

[5]. Comtois, J.H.; Michalicek, M.A.; Barron, C.C. Electro-thermal actuators fabricated in four-level planarized surface micro-machined polycrystalline silicon. Sensors and Actuators A1998, 70, 23-31.

[6]. Spence, John C. H(1988) [1980]. Experimental high-resolution electron microscopy. New York: Oxford U. Press. ISBN 0-19505405-9.

[7]. B.Schaffer, W. Grogger and G. Kothleitner "Automated spatial drift correction for EFTEM image series", Ultra-microscopy, vol. 102, no. 1, pp.27 -36 2004.

[8]. T. Brintlinger, Y. Qi , K. H. Baloch, D. Goldhaber-Gordon and J. Cumings "Electron thermal microscopy", Nano Lett., vol. 8, no. 2, pp.582 -585 2008.

[9]. Sadp Selected area electron diffraction and energy dispersive X-ray analysis for the identification of asbestos fibres, a comparison. Micron (1969) Volume 7, Issue 2, 1976, Pages 115-132 Micron (1969) Volume 7, Issue 2. C.O. Ruud, C.S. Barrett, P.A. Russell, R. L. Clark.

[10]. Selected-area diffraction in the electron microscope. R Phillips.British Journal of Applied Physics, Volume 11, Number 11.

[11]. A software tool for automatic analysis of selected area diffraction patterns within Digital Micrograph ${ }^{\mathrm{TM}}$ C.H. Wua, , , W.T. Reynolds Jr.a, M. Murayamaa, b . Ultramicroscopy.Volume 112, Issue 1, January 2012, Pages 10-14.

[12]. Niladri Halder, Dibyendu Roy: Diagnosis of Computer Assisted Diabetic Retinopathy Using Fundus Image of Retinal Blood Vessels in Macula. International Journal of Current Research Vol. 5, Issue, 08, pp.2156-2160, August, 2013.

[13]. Niladri Halder, Dibyendu Roy, Arnab Chattaraj, Tanumoy Chowdhury: Identification of Cup-Disk Ratio for Glaucoma Prone Eyes. International Journal of Innovative Research in Computer and Communication Engineering Vol. 3, Issue 3, March 2015.

[14]. MehmetSezgin and BulentSankur, Survey over image thresholding techniques and quantitative performance evaluation, Journal of Electronic Imaging 13(1), 146-165 (January 2004). doi:10.1117/1.1631315.

[15]. Bernstein, R.M., K. Edwards and E.M. Eliason, 1975, 'Synthetic Stereo and Landsat Pictures', Photogrammetric Engineering, Vol. 42, pp.1279-1284.

[16]. S. K. Pal and A. Rosenfeld, "Image enhancement and thresholdingbyoptimization of fuzzy compactness,"'PatternRecogn. Lett.7,77-86 1988. 
[17]. Dr. Philippe Cattin (2012-04-24). "Image Restoration: Introduction to Signal and Image Processing". MIAC, University of Basel.Retrieved 11 October 2013.

[18]. Rohankar, Jayant(Nov 2013). "SURVEY ON VARIOUS NOISES AND TECHNIQUES FOR DENOISING THE COLOR IMAGE"(PDF). International Journal of Application or Innovation in Engineering \& Management 2 (11). Retrieved 15 May 2015

[19]. Brink, A.: „Using spatial information as an aid to maximum entropy image threshold selection ${ }^{\text {ee }}$ Pattern Recognit. Lett., 1996, 17, (1), pp. 29-36.

[20]. Johannsen, G., Bille, J.: A threshold selection method using information measures. In: $6^{\text {th }}$ International Conference on Pattern Recognition, pp. 140-143 (1982).

[21]. Kapur, N.J., Sahoo, P.K., Wong, C.K.A.: A new method for gray-level picture thresholding using the entropy of the histogram. J. Comput. Vis. Graph. Image Process.29(3), 273-285 (1985).

[22]. Ab initio phasing of X-ray powder diffraction patterns by charge flipping. Jinsong Wu $\mathbf{u}^{1}$, Kurt Leinenweber ${ }^{2}$, John $\mathrm{C}^{\text {. H. H. Spence }}{ }^{1}$ \& $^{-}$ Michael O'Keeffe ${ }^{2}$. Nature Materials 5, 647 - 652 (2006). Published online: 9 July 2006 doi:10.1038/nmat1687.

[23]. J.1. Br i all , H.E . Kissinger, and L.A. C arlot.In Metastable Materials Form.ltlon by Ion Implantation , ed i ted by S.T. Picrau and W.J. C oyke (Nort h-Rolland, k York, 1982), pp. 235 - 242 P. Moine, M.P. RI fere. N. Junqua, and J. Uelafond. ibid., pp. $243-251$.

[24]. Mogilevsky, P., et al., Evolution of Texture in Rhabdophane-Derived Monazite Coatings. Journal of the American Ceramic Society, 2003.86(10): p. 1767-1772.

[25]. Z. W. Xu\& A. H. W. Ngan (2004): TEM study of electron beam-induced crystallization of amorphous GeSi films, Philosophical Magazine Letters, 84:11, 719-728. 